\title{
Transverse phase space mapping of relativistic electron beams using optical transition radiation
}

\author{
G. P. Le Sage, ${ }^{1}$ T.E. Cowan, ${ }^{1}$ R. B. Fiorito, ${ }^{2}$ and D. W. Rule ${ }^{3}$ \\ ${ }^{1}$ Lawrence Livermore National Laboratory, Livermore, California 94550 \\ ${ }^{2}$ Catholic University of America, Washington, D.C. 20064 \\ ${ }^{3}$ Naval Surface Warfare Center, Carderock Division, West Bethesda, Maryland 20817
}

(Received 8 September 1999; published 20 December 1999)

\begin{abstract}
Optical transition radiation (OTR) has proven to be a versatile and effective diagnostic for measuring the profile, divergence, and emittance of relativistic electron beams with a wide range of parameters. Diagnosis of the divergence of modern high brightness beams is especially well suited to OTR interference (OTRI) techniques, where multiple dielectric or metal foils are used to generate a spatially coherent interference pattern. Theoretical analysis of measured OTR and OTRI patterns allows precise measurement of electron beam emittance characteristics. Here we describe an extension of this technique to allow mapping of divergence characteristics as a function of transverse coordinates within a measured beam. We present the first experimental analysis of the transverse phase space of an electron beam using all optical techniques. Comparing an optically masked portion of the beam to the entire beam, we measure different angular spread and average direction of the particles. Direct measurement of the phase-space ellipse tilt angle has been demonstrated using this optical masking technique.
\end{abstract}

PACS numbers: 29.27.-a, 41.75.Fr, 41.75.Ht

\section{INTRODUCTION}

Transition radiation (TR) is produced by a charged particle as it passes between media with different dielectric constants, for example, a metallic or dielectric foil in vacuum. The angular distribution of this radiation by a single particle reveals details about its energy, position, and direction with respect to the vacuum-foil boundary. An ensemble of particles produces an angular pattern showing the superimposed contributions from each individual particle. By fitting measured angular distributions to theoretical profiles, one can determine statistical beam quality information. For relativistic electron beams, transition radiation can be measured at optical wavelengths (OTR), using readily available equipment and techniques $[1,2]$. When multiple, parallel foils are arranged in the path of a charged particle beam, a spatially coherent OTR interference pattern (OTRI) can be measured [3]. Use of OTRI improves sensitivity of the measurement of beam energy and divergence.

Here we describe the next step in the evolution of OTR emittance diagnostics: the use of OTRI to measure beam divergence as a function of transverse coordinates within the beam profile by use of an optical mask. While the concept of masking imaged OTR light to enable a divergence mapping and phase space reconstruction has been proposed [4], to our knowledge, the present experiment describes the first implementation of alloptical techniques to map beam divergence and direction as a function of transverse position. The OTR method described herein dispenses with the need to introduce apertures into the beam itself.

The traditional phase space mapping technique consists of beam collimation using an array of apertures (a "pepper-pot") or slits [5] to remove the space-charge component of beam-envelope expansion [6]. The emittance induced beamlet expansion is then mapped as a function of drift length and position within the beam. The expansion of each beamlet gives the width of the phase-space ellipse for a given portion of the spatial profile. Spreading of the beamlet centroids also indicates the divergence of the beam at the collimator interface. Examination of several beamlets allows a determination of the CourantSnyder parameters [7] of the transverse phase ellipse.

A basic schematic of a diverging beam producing a low divergence beamlet, with average angle different from that of the whole beam, is shown in Fig. 1. The schematic represents the beam collimation technique for phase space measurement. Scanning the aperture across the beam profile allows mapping of the transverse beam phase space.

By imaging TR light, and masking the light at the image plane, the angular pattern produced by part of the beam can similarly be measured and compared to the angular pattern produced by the entire beam. The imaging technique can be applied to collect data equivalent to a

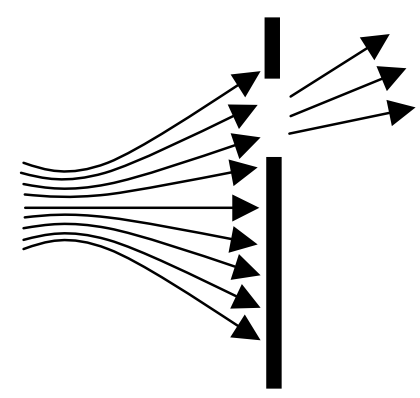

FIG. 1. Schematic of masked beam divergence measurement. 
scanned collimator, while only introducing very thin ( $\mu \mathrm{m}$ scale) foils in the path of the beam.

In addition, since OTR light is radially polarized, a linear polarizer can be used to separately examine orthogonal divergence characteristics of the entire beam or an optically masked beamlet. Two important features can be measured from the OTRI angular pattern: (i) the interference fringe visibility characterizes the divergence distribution width for a given spatial portion of a charged particle beam profile, in analogy to beamlet expansion in the pepper-pot technique; (ii) a centroid shift of the OTRI angular pattern represents a change in average direction for a given beam cross section, in analogy to pepper-pot beamlet separation. Measurement of both the divergence width and divergence centroid as a function of transverse spatial coordinates can be used to completely reconstruct the transverse phase-space ellipse. Here we describe measurement of divergence width and centroid changes as a function of mask position for a relativistic electron beam using optically masked OTRI.

\section{EXPERIMENTAL ARRANGEMENT}

The imaging system used in the present experiment was based on a two-lens telescope, as shown in Fig. 2. Preserving linearity for both position and angle requires a two-lens system in a confocal arrangement: lens spacing is equal to the sum of the lens focal lengths.

The confocal imaging system eliminates the correlation between object position and angular distribution of the OTR in the image plane. A beam profile of arbitrary spatial extent can, in this case, be analyzed with arbitrary image magnification. The OTR image, for all practical purposes, is a magnified version of the light emitted directly by the foil, and its angular distribution is proportional to the original distribution at every point in the image. Masking the light at the image plane is then equivalent to placing a foil with size and position equal to the mask aperture and position within the beam profile.

The OTR imaging system was designed to collect an angular range large in comparison to the characteristic lobe peak separation of $2 / \gamma$. For extracting the divergence information from the entire beam, we require observation of $8 / \gamma(\sim 40 \mathrm{mrad})$ from all points in the beam profile. To achieve this, the following optical system was used. An objective lens (lens 2 in Fig. 2) whose focal length (fl) was $1.22 \mathrm{~m}$ was placed at a distance of $1.26 \mathrm{~m}$ from the second foil of the interferometer. The objective lens diameter was $155 \mathrm{~mm}$, capturing an angular range of $123 \mathrm{mrad}$. First-surface silver mirrors were used to direct this light to a $1.0 \mathrm{~m} \mathrm{fl}$ lens (lens 1 in Fig. 2) placed at a distance of $2.22 \mathrm{~m}$ from the objective lens, corresponding to confocal spacing. The $1.0 \mathrm{~m}$ fl lens had an aperture diameter of $127 \mathrm{~mm}$, reducing the system angular acceptance to $108 \mathrm{mrad}$ from the beam center. The charge-coupled device (CCD) camera used to map the angular OTRI pattern (camera 1 in Fig. 2) had a $96 \mathrm{~mm} \mathrm{fl}$ lens with aperture diameter of $68 \mathrm{~mm}$, focused at infinity. This lens mapped the angle of the incident rays to corresponding positions on a CCD chip. This lens was fixed at a distance of $43 \mathrm{~cm}$ from the mask-image plane. The angular acceptance from the image plane was thus $158 \mathrm{mrad}$. The second lens in the confocal imaging system was thus the limiting aperture, and reduced the acceptance angle down to $108 \mathrm{mrad}$.

The beam spatial distribution at the second foil of the interferometer was imaged at a total distance of $4.45 \mathrm{~m}$ away from the electron beam with an image magnification of 1.22. A mirror-flat aluminum plate with a $4 \mathrm{~mm}$ diameter aperture was used as an optical mask for the phase space mapping experiment. The mask was positioned in the image plane of the second OTR foil, and its aperture defined the portion of the beam whose TR light was used to measure the interference pattern. An optical bandpass filter allows interference fringes to be examined over a specific range of optical wavelengths. The optical bandpass of the system had a $12.7 \%$ FWHM bandwidth centered at $537 \mathrm{~nm}$.

A COHU 6400 series CCD camera was used to collect angular OTRI data. The CCD had $755 \mathrm{H} \times 242 \mathrm{~V}$ active picture elements, with dimensions $6.4 \times 4.8 \mathrm{~mm}(0.5 \mathrm{in}$. format). A frame grabber digitized the interlaced RS 170

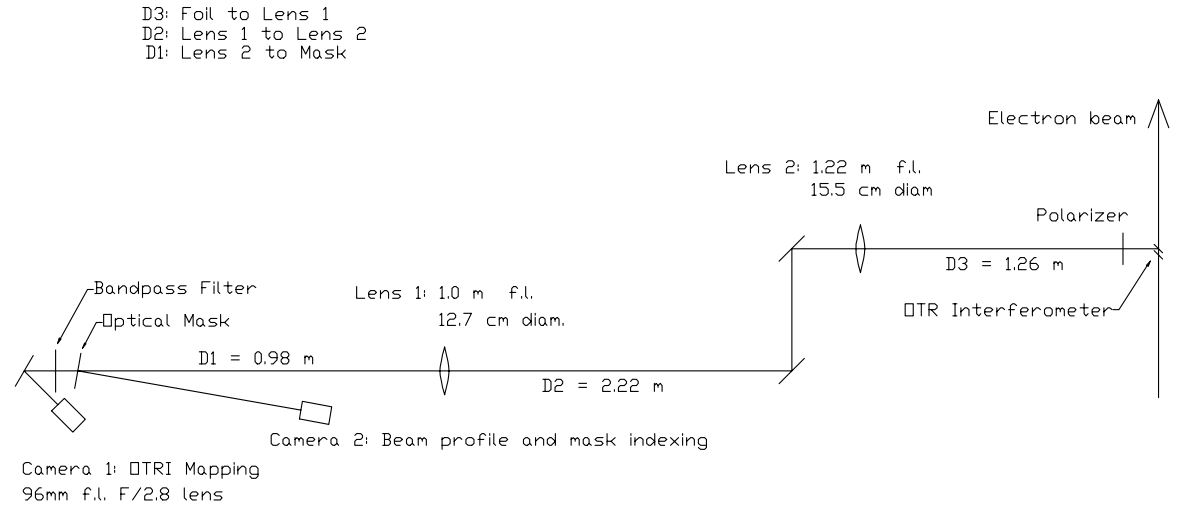

FIG. 2. Masked OTRI experimental arrangement. 
image from the CCD with $640 \mathrm{H} \times 480 \mathrm{~V}$ intensity values. The digitized single "pixel" area is square, with dimensions $10 \times 10 \mu \mathrm{m}$. The term pixel is used in the rest of the discussion to represent one unit area digitized by the frame grabber.

We used an OTR interferometer consisting of a pair of parallel $15 \mu \mathrm{m}$ Mylar ${ }^{\mathrm{TM}}$ foils, aluminized on both sides, and spaced at $25 \mathrm{~mm}$. The foils were oriented at $45^{\circ}$ with respect to the beam direction. Each foil had a diameter of $51 \mathrm{~mm}$, and was stretched over a supporting ring with an outer diameter of $60 \mathrm{~mm}$. A precision machined aluminum block held the foils parallel, and had an exit aperture for OTRI light from the second foil. The acceptance angle of the exit aperture from the center of the second foil was $279 \mathrm{mrad}$ in the horizontal direction and $760 \mathrm{mrad}$ in the vertical direction.

The foil holder was designed so that the limiting aperture was due to the edge of the first foil, not the foil holder. In order to maintain an angular field of view equal to 4 times $1 / \gamma$ on each side of the beam profile, the maximum beam radius must be $3.2 \mathrm{~mm}$, assuming that the profile is centered on the foil support ring. The measured beam profile dimensions were $1.73 \mathrm{~mm}$ horizontally and $2.02 \mathrm{~mm}$ vertically (rms).

The light reflected from the back of the mask consisted of all the light not transmitted through the aperture. This image showed the mask position indexed within the magnified beam profile, and was collected with a separate camera (camera 2 in Fig. 2), focused to image the reflective side of the mask.

\section{MEASUREMENTS}

The intensity profile of OTRI depends on the angle $\theta$ from the specular direction defined by the incident $e^{-}$ trajectory, wavelength $\lambda$, relativistic factor $\gamma$, and foil spacing $L$ through the following simplified relationship:

$$
\frac{\partial^{2} I}{\partial \omega \partial \Omega} \propto \frac{\theta^{2}}{\left(\theta^{2}+\frac{1}{\gamma^{2}}\right)^{2}} \sin \left[\frac{\pi L}{2 \lambda}\left(\theta^{2}+\frac{1}{\gamma^{2}}\right)\right]^{2} .
$$

In the present experiment, the beam energy was 99.6 MeV, corresponding to $\gamma=196$. The energy spread of the beam was $\sigma_{\gamma}=2.7 \mathrm{MeV}$. The $S$-band $\mathrm{rf}$ linac used to produce the beam had a macropulse length of $2.8 \mu \mathrm{s}$, with macropulse current of $69 \mathrm{~mA}$, corresponding to a single macropulse charge of $193 \mathrm{nC}$, at a repetition rate of $10 \mathrm{pps}$.

The optical mask position with respect to the beam profile is shown in Fig. 3. The image is inverted with respect to the light leaving the interferometer foil due to the imaging telescope. The scale is pixel number. Note that the vertical scale is cut in half due to the removal of alternating interlace (zero value) lines.

Approximately $28 \%$ of the magnified electron beam transverse profile image (FWHM area) passed through the mask aperture in this case. The beam profile demonstrates

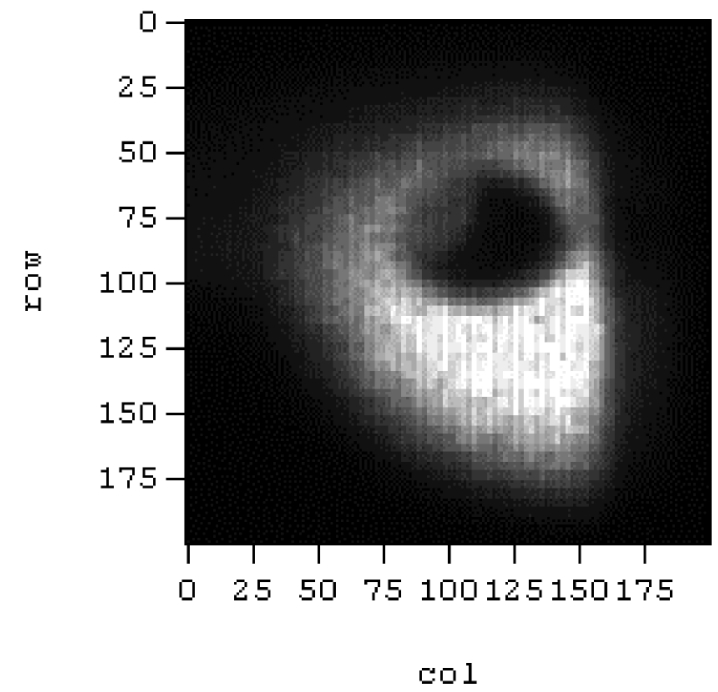

FIG. 3. Deinterlaced image of the electron beam profile with mask aperture.

high order moments in position, which have been routinely observed in our system using conventional profile imaging techniques.

The beam image is magnified by 1.22 with respect to the measured aperture size $(4.0 \mathrm{~mm})$. The beam profile had measured rms widths of $\sigma_{x}=1.73 \mathrm{~mm}$ and $\sigma_{y}=$ $2.02 \mathrm{~mm}$ (demagnified or actual size).

The effective focal length of the OTR imaging system was the camera lens focal length $(96 \mathrm{~mm})$ divided by the imaging magnification (1.22). The angular resolution between the centers of two adjacent $10 \mu \mathrm{m}$ pixels is approximately $0.13 \mathrm{mrad}$. For the vertically polarized OTRI measurements, each data point represents a separation of $20 \mu \mathrm{m}(0.25 \mathrm{mrad})$ due to removal of interlaced (zero value) pixels.

The frame grabber and linac are "line locked" to $60 \mathrm{~Hz}$ ac, allowing the synchronization of the CCD refresh and linac pulses. Each OTRI image presented in the paper represents 127 frames added together, with 127 background frames subtracted. This process was repeated three times, and the resulting images were averaged.

The two-foil OTRI, vertically polarized pattern for the beam core is shown in Fig. 4. The masked images passed less light to the $\mathrm{CCD}$, so the dynamic range was smaller in this case. The normalization of the beam core images consisted of scaling the peak values of the core data with the whole beam data sets. This technique was used to compare lineouts, as in Fig. 6 (below), as well as normalizing Fig. 4 for visual comparison with Fig. 5. Other than this simple scaling, the images were not otherwise processed for presentation. The images in Figs. 4 and 5 were not deinterlaced for presentation. The scale units are pixel number in both cases.

The two-foil OTR interference pattern for the entire beam is shown in Fig. 5. The optical mask was removed from the system in this case. 


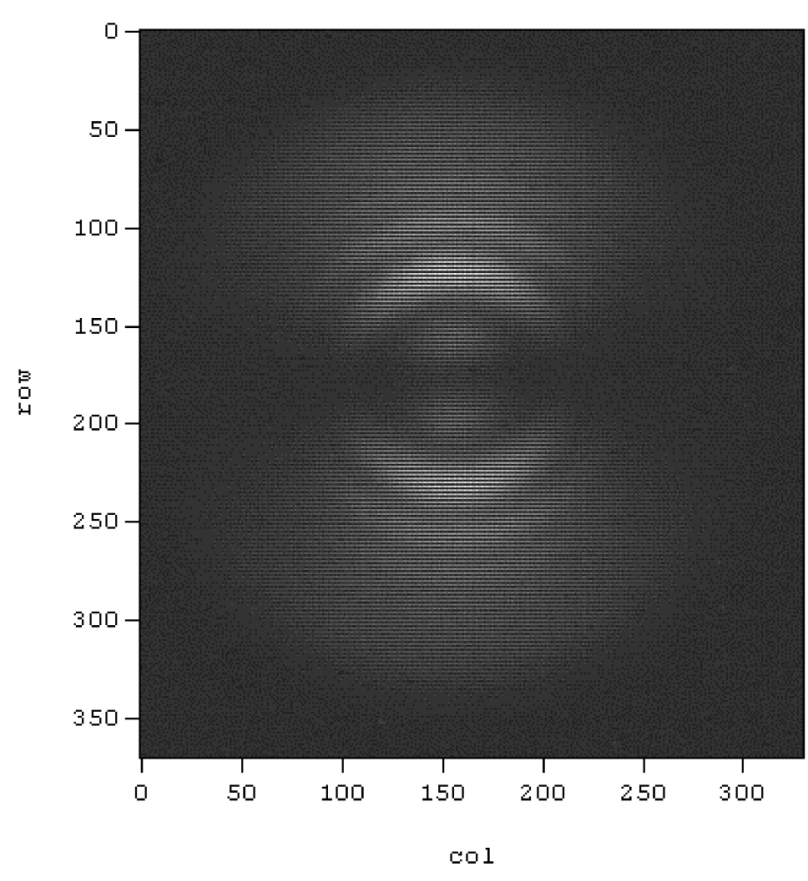

FIG. 4. Beam-core polarized OTR interference pattern.

A qualitative comparison of Figs. 4 and 5 indicates that the beam-core OTRI has sharper lobes than does the whole-beam pattern. The $28 \%$ beam core thus has smaller divergence than does the entire beam.

An important aside to note was that it was possible to diagnose the collimation of the beam at the foil in real time using the visibility of the OTRI fringes. A beam that is collimated at the foil will have maximum interference fringe sharpness. Using a standard monochrome CCD

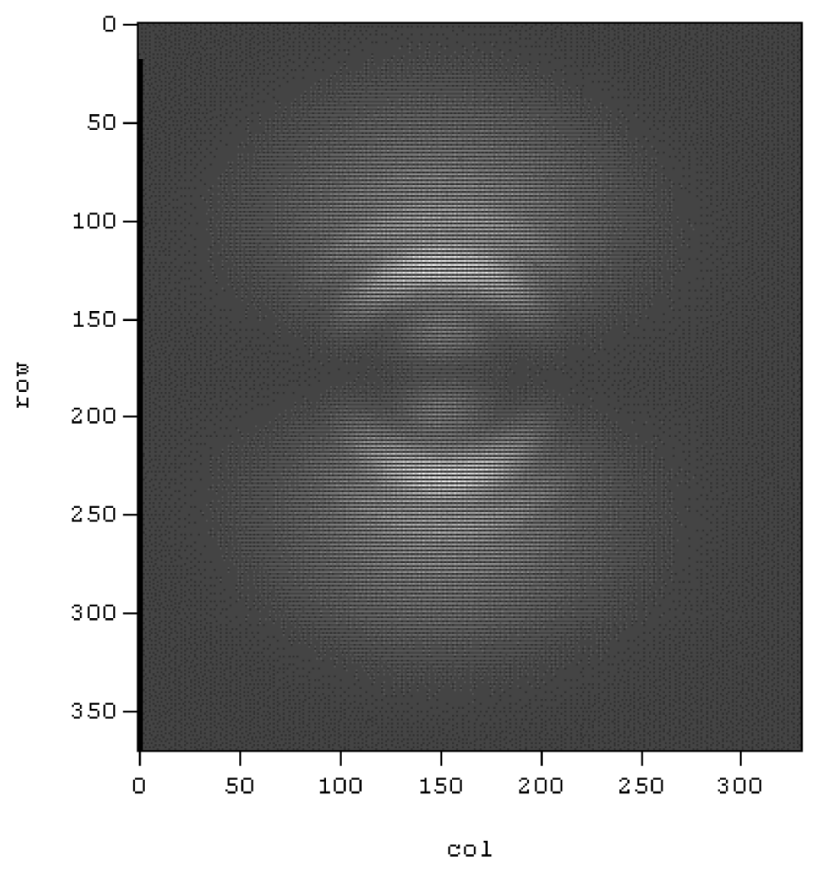

FIG. 5. Whole-beam polarized OTR interference pattern. camera and a triggered frame grabber, the bandpass filtered, polarized OTRI multilobe pattern was visible on a single shot basis. We regularly use this technique to tune the focal parameters of our beam optics, while operating at repetition rates in the range of $1-10 \mathrm{~Hz}$. Since TR also provides the spatial profile of the beam, emittance measurement of the whole beam is straightforward.

With the CCD camera lens focused at infinity, the angular map of the incident rays is then determined by the formula

$$
R_{\mathrm{CCD}}=R_{\mathrm{inc}}^{\prime} E F L,
$$

where $R_{\mathrm{CCD}}$ is the radius on the CCD chip and $R_{\mathrm{inc}}^{\prime}$ is the incident ray direction. A vertical lineout of the OTRI pattern for the whole beam and beam core is plotted in Fig. 6, with the $x$ axis mapped to units of $1 / \gamma$. The intensity scale has arbitrary units in this case.

The lineout comparison results from taking a single column of pixel values from the images shown in Figs. 4 and 5. The interlaced rows (zero value) were removed from the data sets. Three pixel, nearest neighbor smoothing was used in both cases for the presentation of Fig. 6. Qualitative comparison of the OTRI data sets to theoretically generated OTRI profiles indicated a divergence range of $0.6-0.9 \mathrm{mrad}$ for both instances.

A second qualitative feature to note is that the beamcore OTRI angular pattern is offset in angle from the whole-beam profile. This feature implies the ability to distinguish net angular offset of a portion of the beam. The masked portion of the beam has divergence and average direction different from that of the entire beam. We noted this effect qualitatively for several mask positions and measured the offset for one example.

We found that the measured beam was diverging in the vertical direction, and collimated in the horizontal direction, due to the use of the quadrupole focusing with independent coil adjustment. Standard transition radiation analysis of the whole-beam divergence characteristics cannot reveal these details. Imaging and masking of the OTRI light was required to draw these conclusions.

In order to determine the beam divergence, theoretical OTRI distributions based on the measured beam parameters were calculated. Proceeding from the theoretical distribution for a cold beam, or a single particle [Eq. (1)], we generated a normal distribution of divergence angles for a theoretical ensemble of particles, apply a distribution for the known energy spread, and weight several patterns based on the optical bandpass of the system. In order to fit the measured OTRI distributions to theoretical patterns, least-squares fitting was implemented using the two lineouts shown in Figs. 7 and 8.

We estimated the uncertainty associated with each data point in the vertical lineouts by comparing three images taken under identical conditions. Figures 7 and 8 show the average of these three exposures, with the error bars taken from the rms deviation of the exposures from 


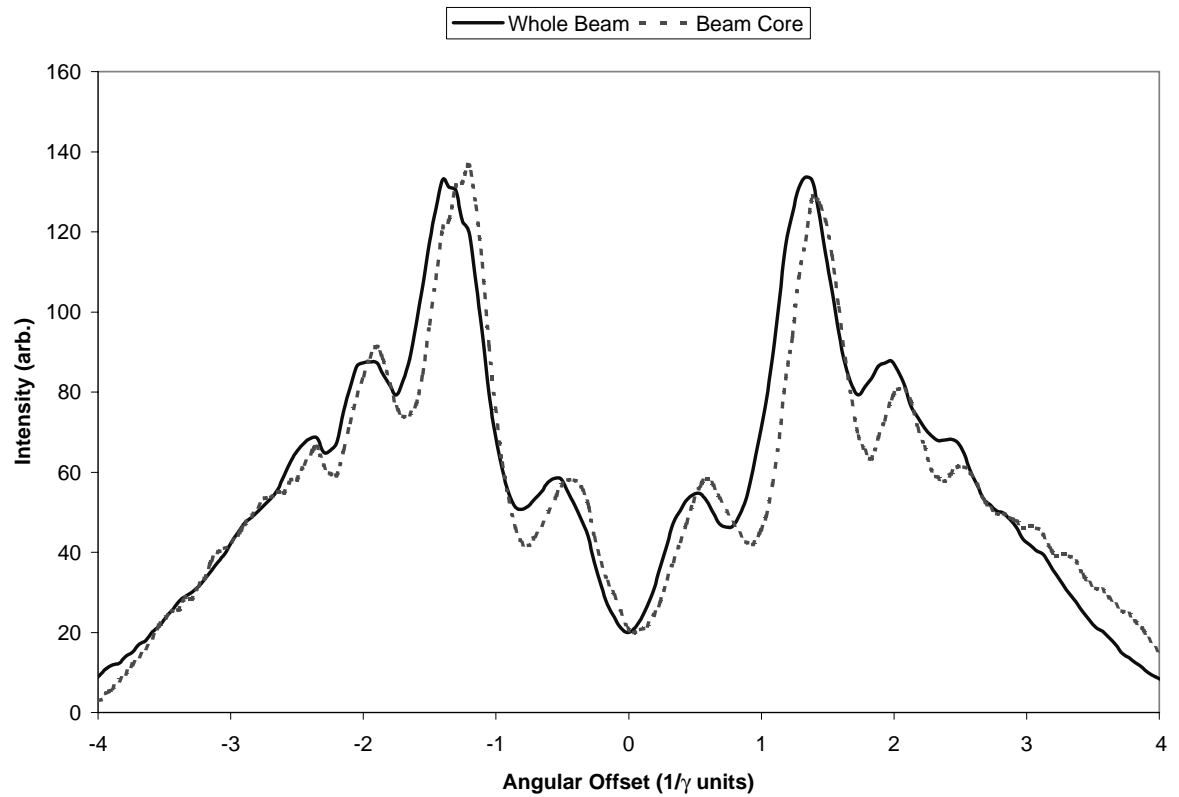

FIG. 6. Lineouts of whole-beam and beam-core vertically polarized OTRI.

this average. The considerably larger error bars for the beam-core data (Fig. 8) reflect the fact that the light intensity was lower, and, hence, there was more noise in the signal above background than for the wholebeam data.

The fit curves in Figs. 7 and 8 are the expected OTRI angular patterns calculated by a Monte Carlo technique, for which we generated 750 beam particle trajectories from an assumed distribution of $\theta_{x}$ and $\theta_{y}$, and from the measured beam energy and optical wavelength distributions. The OTRI intensity in each pixel was calculated by summing over the contributions from each particle, and accounting for the finite pixel size, in addition to the Fresnel reflectivity and optical bandwidth of our filter system. The Monte Carlo generated distribution was then

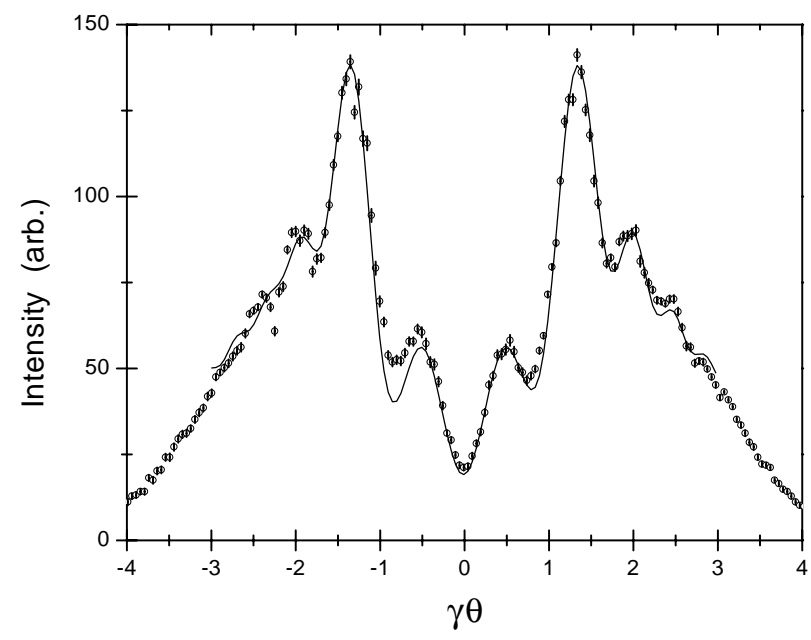

FIG. 7. Whole-beam OTRI fit to $0.85 \mathrm{mrad}$ divergence. compared to the data by adjusting the overall amplitude, the weighted $\chi^{2}$ value calculated, and then the parameters of the input distributions were varied to minimize $\chi^{2}$. The resulting fits were of reasonable quality, and the value of the mean vertical deviation and rms spread in $y^{\prime}$ could be extracted with small uncertainties. The rms $y^{\prime}$ spread was found to be $\sigma_{y^{\prime}}=0.85 \pm 0.02 \mathrm{mrad}$ for the whole-beam distribution and $\sigma_{y^{\prime}}=0.68 \pm 0.02 \mathrm{mrad}$ for the beam core. In addition, the beam-core distribution is shifted from the whole-beam average by a mean value of $y^{\prime}=0.38 \pm 0.02 \mathrm{mrad}$.

The uncertainty in our fitted values in this measurement represents the determination of the angular values to a fraction of a pixel width $(<0.13 \mathrm{mrad})$. This is consistent with usual experience with binned data that one can, for

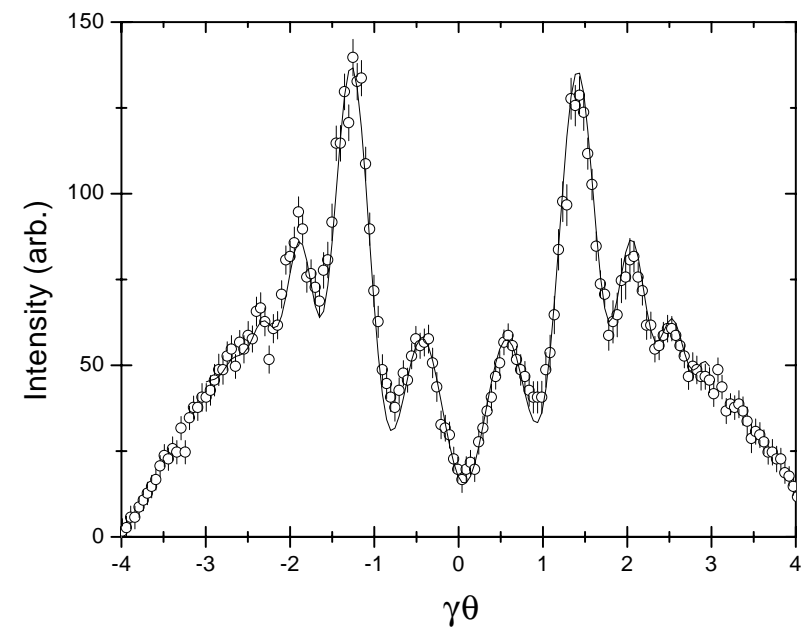

FIG. 8. Beam-core fit to $0.68 \mathrm{mrad}$ divergence. 


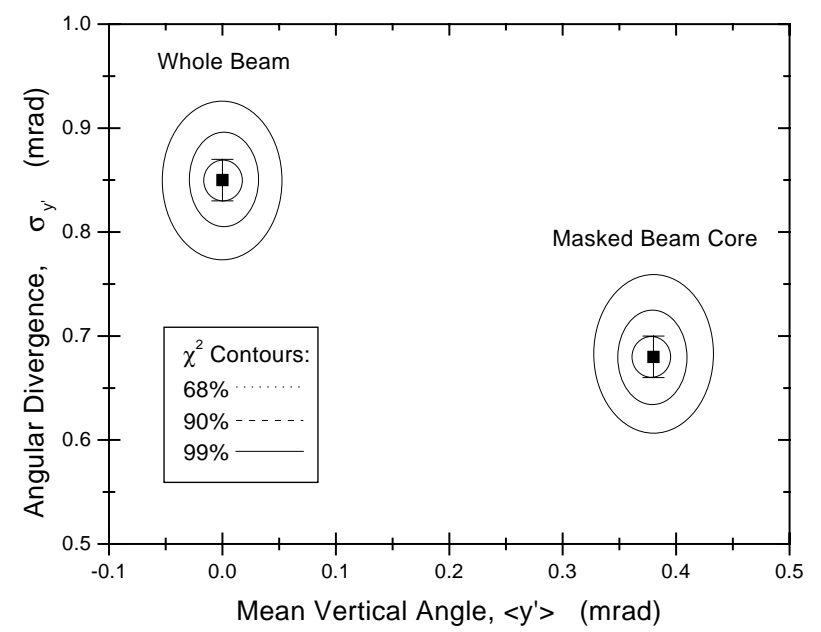

FIG. 9. Contour plot of $\chi^{2}$ values for the divergence and average angle of the whole beam and the core.

example, typically determine a peak position to order of one-tenth of a bin width. We used the likelihood ratio technique to accurately estimate the uncertainty in the best-fit parameters. Figure 9 shows the best fit values and confidence contours for the simultaneously fitted mean divergence angle $y^{\prime}$ and rms angular width $\sigma_{y^{\prime}}$ for the spectra of Figs. 7 and 8.

\section{EMITTANCE DETERMINATION}

These fitted values for the mean $y^{\prime}$ and rms width $\sigma_{y^{\prime}}$, when taken with the spatial data from the mask-indexed beam image (Fig. 3), allow us to determine the phasespace ellipse for the vertical transverse beam emittance. Because the beam is diverging, as indicated by the angular shift in the masked beam-core data, the phase ellipse is tilted, as indicated in Fig. 10. To our knowledge, this is the first measurement of this effect using OTR techniques. Some straightforward improvements are required to develop this into a comprehensive beam emittance diagnostic, but our results indicate the possibility of accurately mapping the entire transverse phase space of an electron beam with a nondestructive, all-optical technique.

One of the limitations of the present measurement is multiscattering of the beam electrons in the first OTR foil, which contributes to the observed angular width. The multiple scattering angle adds roughly in quadrature to the angular width of the assumed normal distribution of incident beam particles,

$$
\theta_{\text {obs }}=\sqrt{\theta_{\text {beam }}^{2}+\theta_{\text {foil }}^{2}} .
$$

The foil-induced divergence angle of the $15 \mu \mathrm{m}$ aluminized Mylar ${ }^{\mathrm{TM}}$ foil, oriented at $45^{\circ}$ to the $100 \mathrm{MeV}$ beam electrons is $0.56 \mathrm{mrad}[2,3]$. By subtracting this value in quadrature from the fitted divergence values, we estimate that the angular divergence values of the incident beam were 0.64 for the whole beam and 0.40 for the beam core.

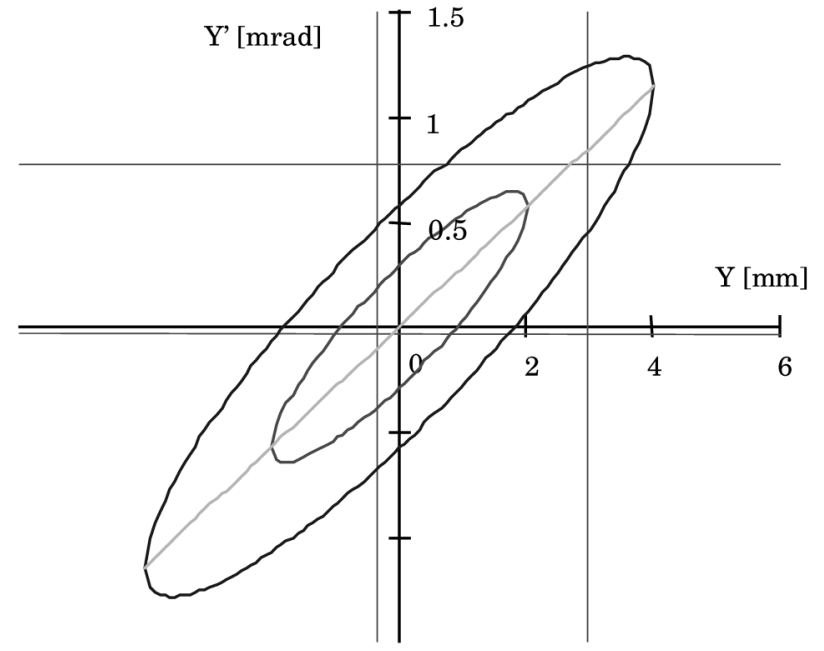

FIG. 10. Estimated phase-space ellipse.

If we had no beam core data, we could, at best, set an upper limit on our beam emittance by multiplying the whole-beam $y$ divergence and rms beam size and obtain a normalized $y$ emittance of

$$
\varepsilon_{y-\mathrm{rms}}^{N}=\gamma \beta \sigma_{y^{\prime}} \sigma_{y}=260 \pi \mathrm{mm} \mathrm{mrad} .
$$

The beam-core data, however, clearly indicate that the beam is diverging in the $y$ direction at the foil position. An estimate of the phase-space ellipse tilt modifies the emittance estimate significantly. The imaged OTRI technique allows calculation of the phase-ellipse tilt angle, demonstrating the key advancement of the experiment.

By way of demonstration, we used the angular offset of the OTRI pattern between the whole beam and beam core to determine Courant-Snyder parameters for the phasespace ellipse. Assuming a linear relationship between spatial mean values and corresponding angular offsets, and the measured values of the beam spatial extent and divergence, the phase-space ellipse can be drawn. The vertical $(y)$ ellipse at $1 \sigma$ and $4 \sigma$ is shown in Fig. 10. The spatial extent of the mask aperture is superimposed (vertical lines), as is the offset divergence width measured for the mask position (horizontal lines). The measured beam correlation slope is drawn through the center of the ellipse.

The Courant-Snyder parameters for the phase-space ellipse consistent with our measured data are $\alpha=-2.0$, $\beta=7.0$, and $\gamma=0.71$.

Since the ellipse tilt is estimated using only a single offset value, the uncertainty in the Courant-Snyder parameters is quite large, nonetheless, in the present measurement we can revise our estimate of the beam emittance by applying the correlation term represented by the ellipse tilt. We find that the phase space area of the beam is 0.45 times smaller (i.e., $\varepsilon^{N} \sim 117 \pi \mathrm{mm}$ mrad) than that calculated above by multiplying the spatial and divergence $\sigma$ widths. While measuring several points in the beam distribution with a smaller aperture will provide a means 
of more accurately mapping the phase-space ellipse, this first demonstration of the technique shows how the additional information provided by an optically masked image can significantly affect beam emittance calculations.

\section{CONCLUSION}

Core and whole-beam divergence has been measured for a $100 \mathrm{MeV}$ thermionic electron beam using imaged OTRI and an optical mask. This allowed a determination of the phase-space ellipse and emittance, without the use of quad-scanning or physical collimation of the beam.

In future work, we will characterize the divergence from an array of mask apertures in a single frame. This will complete the optical analogy to the pepperpot technique. An appropriately spaced linear array of apertures followed by a cylindrical lens positioned at one focal length distance from a CCD array could successfully be used to measure linearly polarized (transverse to the array direction) OTR light simultaneously from separate portions of a beam profile

In addition to providing divergence information, we found that OTRI provided a sensitive measure of the beam energy, particularly in the fractional order (central) lobes. Here the precise phase of the interference term in Eq. (1) results in large amplitude changes for small (percent level) changes in beam energy. Similar behavior has been noted and exploited in the past by other groups [3]. In principal, this effect may be used to determine spatial energy correlations. With the addition of a timeresolved measurement of the intensity of various portions of the OTRI pattern, it may be possible to map time-slice emittances as well as longitudinal beam emittance.

\section{ACKNOWLEDGMENTS}

We would like to acknowledge the expert assistance of G. G. Anderson, R. P. Hall, and W.S. Patterson in fielding these experiments. This work was performed under the auspices of the U.S. Department of Energy by the Lawrence Livermore National Laboratory under Contract No. W-7405-Eng-48.

[1] Ralph B. Fiorito and Donald W. Rule, in Beam Instrumentation Workshop, edited by R. E. Shafer, AIP Conf. Proc. No. 319 (AIP, New York, 1994).

[2] D. W. Rule, Nucl. Instrum. Methods Phys. Res., Sect. B 24/25, 901 (1987).

[3] L. Wartski, S. Roland, J. Lasalle, M. Bolore, and G. Filippi, J. Appl. Phys. 46, 3644 (1975).

[4] Ralph B. Fiorito and Donald W. Rule, US Patent No. 5120968, 1992.

[5] J. Rosenzweig and G. Travish, http://pbpl.physics.ucla. edu/papers/

[6] It should be noted that "quad-scan" techniques measure only the whole-beam average emittance values and are affected by space charge. There is no ability to raster through individual sections of the beam, and beam statistics must be gathered over a large number of pulses as the magnet parameters are varied. Transition radiation techniques are unaffected by space charge considerations. See Helmut Wiedemann, Particle Accelerator Physics: Basic Principles and Linear Beam Dynamics (SpringerVerlag, New York, 1993).

[7] E. D. Courant and H.S. Snyder, Ann. Phys. 3, 1-48 (1958). 